\title{
Laser-Photofield Emission from Needle Cathodes for Low-Emittance Electron Beams
}

\author{
R. Ganter, R. Bakker, C. Gough, S. C. Leemann, M. Paraliev, M. Pedrozzi, F. Le Pimpec, \\ V. Schlott, L. Rivkin, and A. Wrulich \\ Paul Scherrer Institute, 5232 Villigen, Switzerland \\ (Received 24 August 2007; published 13 February 2008)
}

\begin{abstract}
Illumination of a $\mathrm{ZrC}$ needle with short laser pulses $(16 \mathrm{ps}, 266 \mathrm{~nm})$ while high voltage pulses $(-60 \mathrm{kV}, 2 \mathrm{~ns}, 30 \mathrm{~Hz})$ are applied, produces photo-field emitted electron bunches. The electric field is high and varies rapidly over the needle surface so that quantum efficiency $(\mathrm{QE})$ near the apex can be much higher than for a flat photocathode due to the Schottky effect. Up to $150 \mathrm{pC}$ (2.9 A peak current) have been extracted by photo-field emission from a $\mathrm{ZrC}$ needle. The effective emitting area has an estimated radius below $50 \mu \mathrm{m}$ leading to a theoretical intrinsic emittance below $0.05 \mathrm{~mm} \mathrm{mrad}$.
\end{abstract}

The production of short electron bunches with lower emittance and higher brightness than the state-of-the-art would have consequences in a variety of scientific and industrial applications [1]. It is the most direct way to reduce the cost and size of Free Electron Lasers (FELs) [2]. In linear accelerators, the electron beam properties at high energies depend strongly on the emittance of the electron source $[3,4]$. A field emitted electron beam has an energy spread around $0.3 \mathrm{eV}$ [5] and can reach very high intensity (up to $10^{12} \mathrm{~A} \mathrm{~m}^{-2}$ for field emission [6] in comparison to $10^{9} \mathrm{~A} \mathrm{~m}^{-2}$ [7] for photoemission). Since the source emittance is proportional to the emitting area size, field-emitter sources can potentially produce much lower emittance than photoemission sources [8,9]. The main drawback of field emitters like needle cathodes is the risk of destructive arc formation when one tries to extract large currents. The use of high voltage pulsed generators (in the nanosecond range) at low repetition rates significantly reduces the heating of the tip and the related risk of breakdown $[10,11]$. In addition, the illumination of a needle cathode with short laser pulses (in the picoseconds range) while field emission occurs, should produce a photoemission pulse with a duration equal to the laser pulse length on the top of the field emission pulse [12,13]. In this Letter, we would like to show the possibility of extracting peak currents of several amperes by photo-field emission on a needle cathode. Such peak currents are close to the requirements $(5.5$ A) for the X-ray FEL concept of the Paul Scherrer Institute [14]. The electron emission is confined to a small area near the needle apex where quantum efficiency $(\mathrm{QE})$ is very high $[15,16]$. In consequence, the intrinsic emittance can be much smaller than for a flat photocathode. Sources of emittance growth after the emission are not investigated in this work. However, preliminary measurement of emittance with a nonshielded solenoid (magnetic field was present near the source) gives an upper limit of the expected emittance.

In this experiment, we synchronized a $\mathrm{Nd}$ :vanadate (Nd:VAN) mode locked picosecond laser system (longitu- dinal Gaussian distribution $\sigma_{t, \text { laser }}=16 \mathrm{ps} \mathrm{rms}, \quad \lambda=$ $266 \mathrm{~nm})$ [17] with a fast high voltage pulser $(60 \mathrm{kV}, 2 \mathrm{~ns}$ [18]) to measure the charge and the transverse properties of photo-field-emitted electron bunches from a $\mathrm{ZrC}$ needle (see Fig. 1). The energy per pulse can reach $20 \mu \mathrm{J}$ at maximum and the transverse laser intensity profile at the position of the cathode $I_{\text {laser }}(\rho)$ is Gaussian with an rms width $\sigma_{r \text {,laser }}$. The pulser can produce negative voltage pulses of $2 \mathrm{~ns}$ FWHM with amplitudes $V_{\text {Tip }}$ up to $60 \mathrm{kV}$ at maximum and with rise/fall time of $1 \mathrm{~ns}$ and a time jitter of $200 \mathrm{ps}$ from pulse to pulse. The $\mathrm{ZrC}$ emitter needles were produced by etching ultrapure $\mathrm{ZrC}$ single crystal rods of $500 \mu \mathrm{m}$ diameter $[19,20]$. For the measurements described below, all the photons are intercepted by the needle rod. As received, the needle apex radius $r_{\text {apex }}$ is less than $1 \mu \mathrm{m}$ but we voluntarily blunt the apex to several micrometers in order to sustain field emission pulses of several tens of $\mathrm{mA}$ at $30 \mathrm{kV}$ (initial conditioning period) [11]. A Faraday cup (6 GHz Bandwidth) is placed $20 \mathrm{~cm}$ away from the cathode and connected to a $1 \mathrm{GHz}$ bandwidth scope. A solenoid ( $1 \mathrm{~cm}$ thick) is positioned at about $2 \mathrm{~cm}$ in front of the tip and used in order to focus the electron

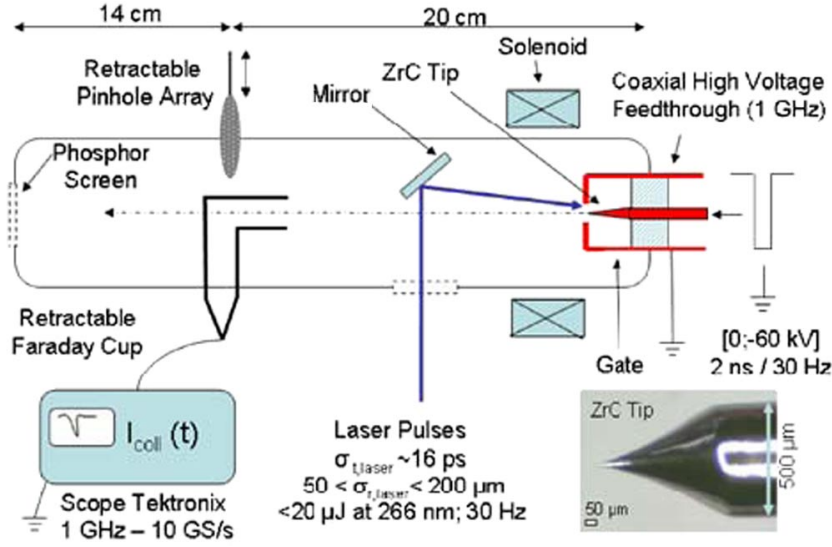

FIG. 1 (color online). Experimental Setup used for photo-field emission tests; Inset: Micrograph of a $\mathrm{ZrC}$ needle. 
beam on the Faraday cup $(20 \mathrm{mT}$ per solenoid at maximum). The needle was not shielded from magnetic field lines. The Faraday cup can be replaced by a pinhole array for emittance measurements. The beamlets are intercepted by a phosphor screen $14 \mathrm{~cm}$ downstream of the pinhole array. The needle is directly attached to the inner conductor of a custom designed high voltage coaxial vacuum feedthrough (bandwidth $\sim 1 \mathrm{GHz}$ ). Since the transmission line is not properly terminated (open circuit at the end of the needle), there is a strong positive reflection of the applied negative voltage pulse as can be seen on the voltage waveform in Fig. 2. A telescope lens assembly is used to adjust the laser spot size on the needle apex. The laser power density is optimized in order to stay below the explosive electron emission (EEE) regime [21-23] but still high enough to provide a maximum number of photons on the apex. A safe laser power density was found to be $\sim 0.1 \mathrm{GW} / \mathrm{cm}^{2}$ at $266 \mathrm{~nm}$ and $50 \mathrm{kV}$. Above $1 \mathrm{GW} / \mathrm{cm}^{2}$, the risks of EEE becomes too important as it can be observed in photoguns [24].

Figure 2 (top) shows the field emission current extracted from the $\mathrm{ZrC}$ needle, when a (1.5 ns FWHM) high voltage pulse is applied. As soon as laser pulses illuminate the needle, in presence of high voltage, the current pulse amplitude increases (Fig. 2 bottom) with an apparent duration shorter than $1 \mathrm{~ns}$, that is to say below the resolution of the scope. The time profile of the photocurrent pulses should follow the laser pulse shape, but the bandwidth of the diagnostic system (scope and Faraday cup) does not allow the observation. However, the integral of the waveform is real and corresponds to $56 \mathrm{pC}(=72 \mathrm{pC}-16 \mathrm{pC})$ of photo emitted charge. This corresponds to an emitted peak current around 1.4 A. Peak currents as high as $2.9 \mathrm{~A}$ were obtained at $V_{\text {Tip }}=-59 \mathrm{kV}$ (see Fig. 3, top).

In Fig. 3, one can see the effect of solely increasing the bias voltage on the needle: the QE increases with the needle voltage. This can be explained with the Fowler-

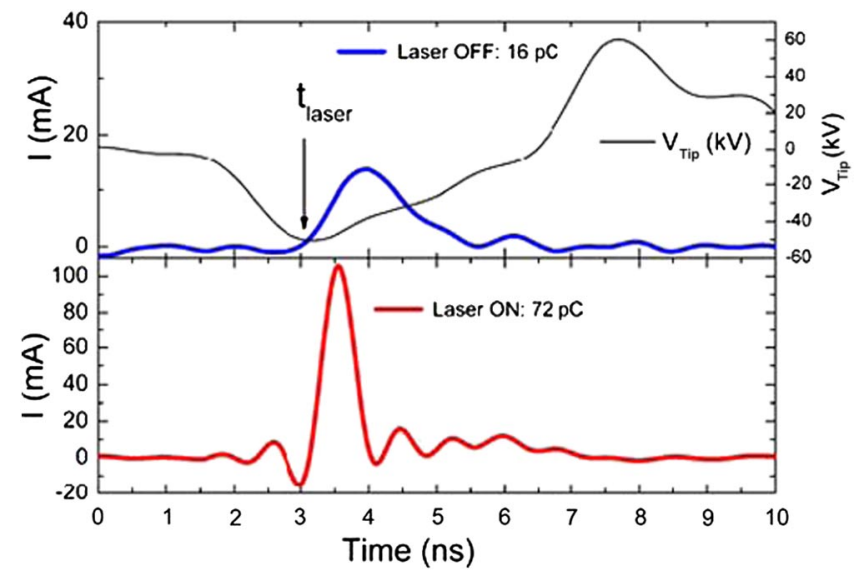

FIG. 2 (color online). Measured current pulses when high voltage pulses are applied to a $\mathrm{ZrC}$ needle with and without laser illumination $\left(266 \mathrm{~nm} ; \sigma_{t \text {, laser }}=16 \mathrm{ps} ; \sigma_{r \text {, laser }}=85 \mu \mathrm{m}\right.$; $\left.E_{266 \mathrm{~nm}}=6 \mu \mathrm{J} ; 30 \mathrm{~Hz}, V_{\text {Tip }}=-45 \mathrm{kV}\right)$.
Dubridge model [25] in which the emission probability increases with the surface electric field. The barrier height that retains electrons in the conduction band decreases with the applied electric field so that the probability of having photo-excited electrons above the barrier height increases $[13,15,26]$. If one considers the uppermost plot of Fig. 3 $\left(E_{266 \mathrm{~nm}}=6 \mu \mathrm{J}\right.$ of photons producing $144 \mathrm{pC}$ ), the apparent $\mathrm{QE}$ of the $\mathrm{ZrC}$ tip is around $10^{-4}$. However, this QE represents only an average of the QE distribution over the tip surface. The electric field rapidly decreases down the sides of a needle (field enhancement effect), and the laser energy is less absorbed because of a less favorable angle of incidence [15]. This is illustrated by Fig. 4 which shows that the number of collected photo electrons steeply varies with laser spot radial position $\rho_{\text {laser }}$ (relative to needle axis) while retaining the same number of photons per laser pulse. In order to estimate the emitting area, we assumed an exponential decay of the QE with the radial position over the needle surface $[\mathrm{QE}(\rho)=$ $\left.\mathrm{QE}_{0} \exp \left(-\rho / \rho_{0}\right)\right]$. Such distribution is only a rough approximation of the more detailed description from Jensen et al. [15]. The emitted charge can then be expressed by the following expression:

$$
Q=\frac{e \lambda}{h c} \int_{0}^{2 \pi} \int_{0}^{+\infty} I_{\text {laser }}\left(\rho-\rho_{\text {laser }}\right) \times \mathrm{QE}(\rho) d \theta d \rho .
$$

A good agreement is obtained between the measured data and the fit for $\mathrm{QE}_{0}=10^{-2}$ and $\rho_{0}=2 \mu \mathrm{m}$. Such

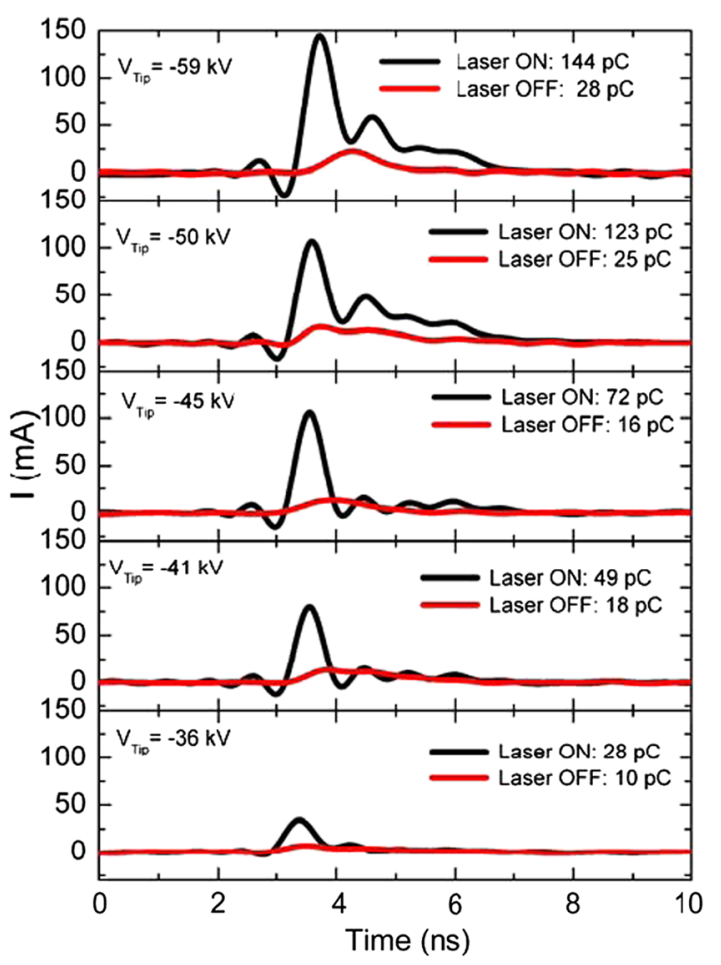

FIG. 3 (color online). Current waveforms collected for different $V_{\text {Tip }}$, with and without laser illumination; Laser: $266 \mathrm{~nm}$, $E_{266 \mathrm{~nm}}=6 \mu \mathrm{J}, \sigma_{z \text {, laser }}=16 \mathrm{ps}, \sigma_{r, \text { laser }}=85 \mu \mathrm{m}$. 


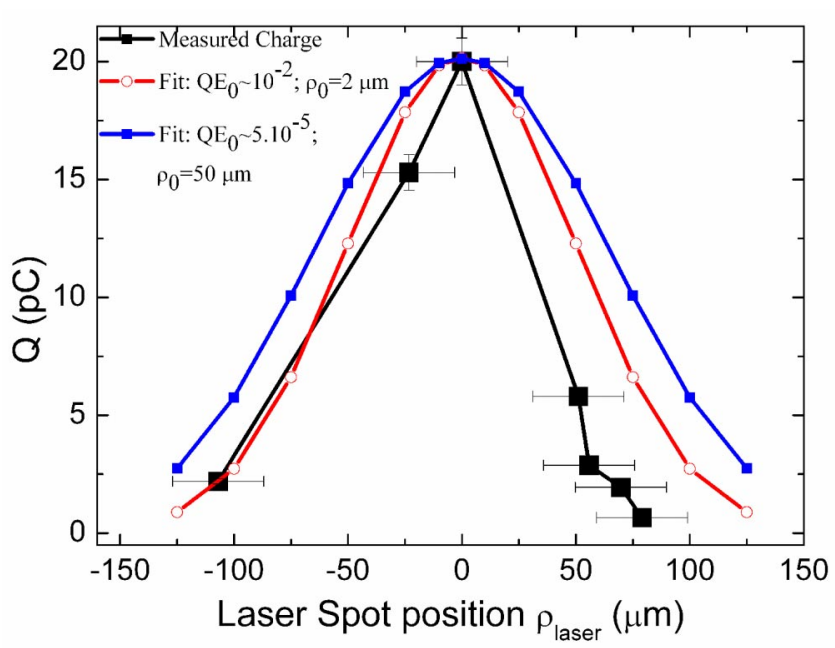

FIG. 4 (color online). Emitted charge (average over 20 acquisitions) versus laser spot radial position $\rho_{\text {laser }}$ (the zero corresponds to the needle axis), $E_{266 \mathrm{~nm}}=3 \mu \mathrm{J}, \sigma_{z \text {,laser }}=16 \mathrm{ps}$ $(\mathrm{rms}), \sigma_{r \text { laser }}=50 \mu \mathrm{m}(\mathrm{rms}), V_{\text {Tip }}=-32 \mathrm{kV}$. Theoretical fit corresponds to Eq. (1) with $\mathrm{QE}(\rho)=\mathrm{QE}_{0} \exp \left(\rho / \rho_{0}\right)$.

distribution implies that $\mathrm{QE}$ becomes negligible for $\rho>$ $50 \mu \mathrm{m}$ so that the effective emitting area is the surface of the needle apex with $\rho<50 \mu \mathrm{m}$.

The photo-field-emitted current is also very sensitive to the delay $\Delta t$ between the laser pulse and the instant of maximum voltage. The time of flight of electrons from the needle to the Faraday cup is about $1 \mathrm{~ns}$ at $39 \mathrm{keV}$. When the laser strikes the needle $0.6 \mathrm{~ns}$ before the maximum voltage pulse (Fig. $5, \Delta t=-0.6 \mathrm{~ns}$ ), only a small effect is observed on the collected field emitted current pulse (the photoelectrons are emitted before $V_{\text {Tip }}$ reaches his maximum value). A strong increase in the collected current is observed when the laser pulse is synchronized with the instant of the maximum voltage pulse $(\Delta t=0 \mathrm{~ns}$, maximum Schottky effect). The photoemission component of the current decreases again when the laser illumination occurs during the fall of the high voltage pulse $(\Delta t>$ $0 \mathrm{~ns}$ ). By changing $\Delta t$, one can illuminate the needle at various $V_{\text {Tip }}$ such that photoelectrons do have different energies than field emitted electrons.

The advantage of photo-field emission in comparison to standard photoemission is that high electric field (a few $\mathrm{GV} / \mathrm{m}$ ) is present over a small area near the needle apex leading to a much higher QE locally. The emitting area radius can then be reduced to get smaller intrinsic emittance [8,27]. Typical photoguns with copper photocathodes exhibit intrinsic emittance around $10^{-6} \mathrm{~m} \mathrm{rad}$ with an emitting area radius of $1 \mathrm{~mm}$ [28-30]. With an emitting area radius of $\sim 50 \mu \mathrm{m}$ (as mentioned above), the intrinsic emittance could be as small as $5.10^{-8} \mathrm{~m}$ rad. In the following measurements, we have tried to measure the rms emittance from the needle photo-field beam. Magnetic field lines from the solenoid (see Fig. 1) were probably present around the needle, introducing some emittance

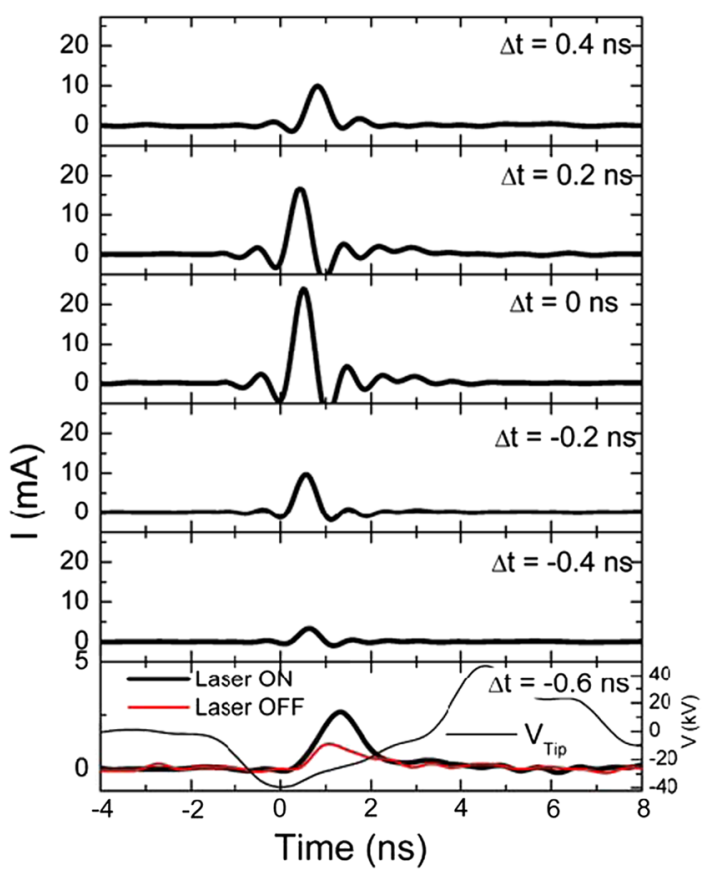

FIG. 5 (color online). Collected current pulses (single acquisitions) for various delay $\Delta t$ between the instant of laser illumination and the voltage pulse maximum ( $V_{\text {Tip }}=-39 \mathrm{kV}$, $E_{266 \mathrm{~nm}}=6 \mu \mathrm{J}, \sigma_{r, \text { laser }}=85 \mu \mathrm{m}, 30 \mathrm{~Hz}$ ).

growth just after emission. Nevertheless, this emittance measurement gives an upper limit for such electron source. Figure 6 represents an image of the photo-field emitted electron beam projected on a phosphor screen.

To measure the transverse rms emittance (as defined in [31]), we focused the beam on a pinhole array inserted between the phosphor screen and the needle cathode (see Fig. 1). Since the magnetic field was affecting the beam emittance, no accurate emittance measurements were possible for energies above $30 \mathrm{keV}$.

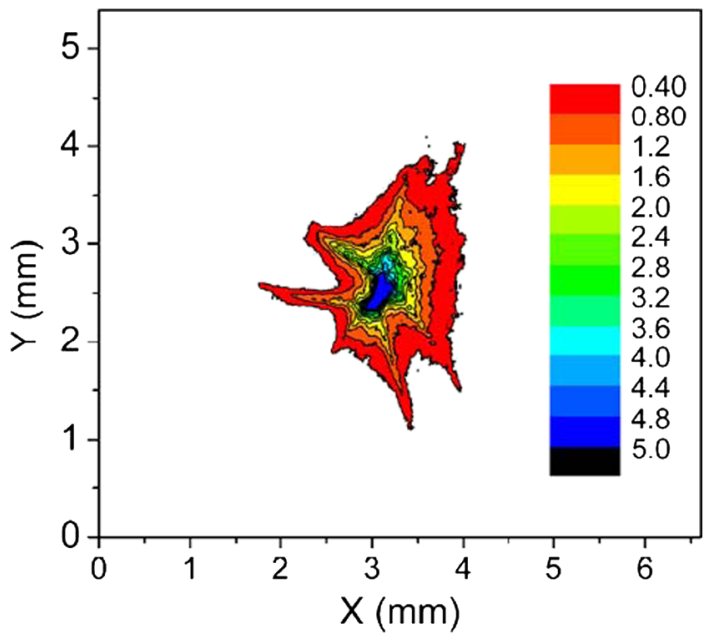

FIG. 6 (color online). Photo-field emitted beam spot on a phosphor screen (43 keV; $50 \mathrm{pC} ; 30 \mathrm{~Hz}$ ). 

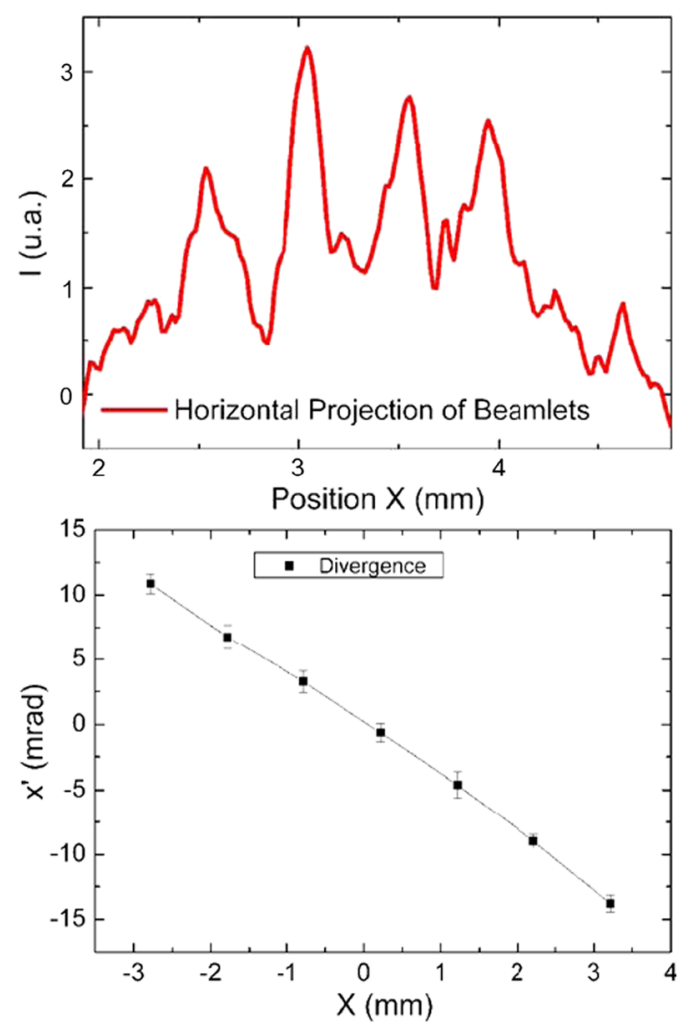

[1] B. J. Claessens et al., Phys. Rev. Lett. 95, 164801 (2005).

[2] R. J. Bakker et al., Proceedings of the FELO6 Conference (BESSY, Berlin, Germany, 2006).

[3] V. Miltchev et al., Proceedings of the 27th FEL Conference (SLAC, Menlo Park, California, 2005), p. $560-563$.

[4] J.E. Clendenin et al., Nucl. Instrum. Methods Phys. Res., Sect. A 455, 198 (2000).

[5] R. D. Young, Phys. Rev. 113, 110 (1959).

[6] G. Fursey, Field Emission in Vacuum Microelectronics (Plenum Publishers, New York, 2005).

[7] R. Bossart et al., Proceedings of the Particle Accelerator Conference (SLAC, Menlo Park, California, 1995), p. 719.

[8] K. L. Jensen et al., Appl. Phys. Lett. 89, 224103 (2006).

[9] J. F. Schmerge et al., Report No. SLAC-PUB-11700, 2006.

[10] D. V. Glazanov, L. M. Baskin, and G. N. Fursey, Sov. Phys. Tech. Phys. 34, 534 (1989).

[11] R. Ganter et al., Nucl. Instrum. Methods Phys. Res., Sect. A 565, 423 (2006).

[12] M. Boussoukaya et al., Nucl. Instrum. Methods Phys. Res., Sect. A 279, 405 (1989).

FIG. 7 (color online). Horizontal projection of the pinhole image transverse profile (left), beamlet divergence centroids versus position centroids (right) $(27 \mathrm{keV} ; 20 \mathrm{pC} ; 266 \mathrm{~nm}$; $E_{266 \mathrm{~nm}}=2.3 \mu \mathrm{J} ; \sigma_{t, \text { laser }}=16 \mathrm{ps} ; 30 \mathrm{~Hz}$ ).

Figure 7 (top) represents the projections of beamlet's intensities in the horizontal direction for a bunch of $20 \mathrm{pC}$ at $27 \mathrm{keV}$. With the calculation of the beamlet divergence versus the position centroids (Fig. 7, bottom), one can deduce the rms projected beam emittance [27,32]. Taking into account the weak signal to noise ratio and the beamlet overlap, one can estimate a normalized rms projected emittance between 0.3 and $0.7 \mathrm{~mm}$ mrad. The magnetic field at the cathode might be the major source of emittance dilution because of the short solenoid to needle distance. The analyses of the different sources of emittance increase go beyond the scope of this Letter. The above measured value gives an upper limit for the rms normalized emittance.

The laser illumination of a needle cathode emitting moderate field emission current $(\mathrm{mA})$ produces short electron bunches with high current density. Peak currents up to 2.9 A ( $144 \mathrm{pC}$ in $16 \mathrm{ps}$ rms pulses) have been extracted from a $5 \mu \mathrm{m}$ tip radius. Considering the highly nonuniform $\mathrm{QE}$ distribution over the tip surface, the emitting area radius is estimated to be below $50 \mu \mathrm{m}$ leading to an intrinsic emittance of $5.10^{-8} \mathrm{mrad}$. A normalized projected emittance between 0.3 and $0.7 \mathrm{~mm}$ mrad has been measured at low bunch charge $(20 \mathrm{pC})$ without shielding the needle from magnetic fringe fields. Further improve-

[13] C.H. Garcia and C.A. Brau, Nucl. Instrum. Methods Phys. Res., Sect. A 483, 273 (2002).

[14] http://fel.web.psi.ch.

[15] K. L. Jensen, D. W. Feldman, and P. O'Shea, J. Vac. Sci. Technol. B 23, 621 (2005).

[16] J. D. Zuber, K. L. Jensen, and T. E. Sullivan, J. Appl. Phys. 91, 9379 (2002).

[17] http://www.tbwp.com.

[18] http://www.fidtechnology.com/.

[19] F. M. Charbonnier et al., J. Vac. Sci. Technol. B 19, 1064 (2001).

[20] http://www.a-p-tech.com/.

[21] G. A. Mesyats, Plasma Phys. Controlled Fusion 47, A109 (2005).

[22] G. N. Fursey et al., Appl. Surf. Sci. 215, 286 (2003).

[23] R. Ganter et al., Proceedings of the FELO6 Conference (BESSY, Berlin, Germany, 2006).

[24] X. J. Wang et al., J. Appl. Phys. 72, 888 (1992).

[25] L. A. Dubridge, Phys. Rev. 43, 727 (1933).

[26] T. Srinivasan-Rao, J. Fischer, and T. Tsang, J. Appl. Phys. 69, 3291 (1991).

[27] S. Humphries, Charged Particle Beams (John Wiley \& Sons, New York, 1990).

[28] V. Miltchev et al., Proceedings of the FEL Conference (Comitato Conferenze Elettra, Trieste, Italy, 2004), p. 399-402.

[29] R. Akre et al., Proceedings of the PAC07 Conference (Albuquerque, New Mexico, USA, 2007).

[30] J.F. Schmerge et al., Nucl. Instrum. Methods Phys. Res., Sect. A 483, 301 (2002).

[31] M. Reiser, Theory and Design of Charged Particle Beams (John Wiley \& Sons, New York, 1994).

[32] S. C. Leemann, A. Streun, and A. F. Wrulich, Phys. Rev. ST Accel. Beams 10, 071302 (2007). 